\title{
Expression of CD133 Correlates with Tumor Stage, Lymph Node Metastasis and Recurrence in Oral Squamous Cell Carcinoma \\ Reggiani Bonetti $L^{1}$, Migaldi $\mathbf{M}^{1}$, Boninsegna $A^{2}$, Fanali $C^{2}$, Farina $\mathbf{M}^{2}$, Chiarini $L^{3}$, Anesi $A^{3}$, Cittadini $A^{2}$, Leocata $P^{4}$, Maccio $L^{1}$ and Sgambato $A^{2 *}$
}

${ }^{1}$ Dipartimento di Laboratori, Anatomia Patologica e Medicina Legale, University of Modena and Reggio Emilia, Modena, Italy

${ }^{2}$ Istituto di Patologia Generale, Università Cattolica del Sacro Cuore, Rome, Italy

${ }^{3}$ Dipartimento di Chirurgie specialistiche testa - collo, University of Modena and Reggio Emilia, Modena, Italy

${ }^{4}$ Dipartimento di Anatomia Patologica, University of L'Aquila, L'Aquila, Italy

\begin{abstract}
Objective: CD133 is a pentaspan membrane protein expressed by the so-called cancer stem cells (CSC) in the majority of human cancers. The aim of this study was to analyze CD133 expression levels in specimens of oral cancer and to evaluate its relation with disease evolution.
\end{abstract}

Methods: Expression of the CD133 protein was evaluated by immunostaining in a series of oral squamous cell carcinoma (OSCC) and its relation with traditional prognostic indicators and the clinical outcome of patients was analyzed.

Results: CD133 expression was highly variable amongst different samples with a median percentage of positive cells of 5 (range $0-80$; mean $=11$ ) and CD133 staining was not detectable in tumor cells in $29(43 \%)$ cases. No correlation was observed with age at diagnosis, gender and grading while a significant correlation was observed with tumor stage. Kaplan-Meier curves of disease-free survival displayed a significant separation between the negative and positive groups of patients ( $p=0.001$ by log-rank test) but CD133 staining did not confirm to be an independent predictor of clinical outcome in a multivariate analyses.

Conclusion: Expression of CD133 was detectable in the majority of OSCC samples and correlated significantly with tumor stage and the clinical outcome of patients in terms of disease-free survival. Further studies are warranted on a larger series of cases to elucidate the role of CD133 in the development and progression of OSCC and its suitability as a prognostic biomarker.

Keywords: Oral squamous carcinoma; CD133; Prognostic marker; Oral cancer; Tumorigenesis

\section{Introduction}

Oral cancer is the most frequent cancer of the head and neck region with oral squamous cell carcinomas (OSCC) accounting alone for about $90 \%$ of all malignancies of the oral cavity [1]. OSCC is regarded as a serious health problem world-wide due to its high mortality and low rate of cure. In fact, the five-year survival rate has remained at a disappointingly stable level, almost unchanged over the past 20 years [1]. Moreover, OSCC can display a highly variable biological and clinical behavior which cannot be accurately predicted by the available prognostic markers (i.e., tumor stage and grade). Thus, a great effort is devoted to identifying new prognostic indicators for OSCC patients and several molecular promising biomarkers have been identified [2]. These markers relate to major biological pathways such as control of cell cycle and growth, cell survival and programmed cell death, invasion and angiogenesis but none of them has yet been routinely adopted in the clinic for patient management [3].

Recent evidence have suggested that tumors, like normal tissues, display a hierarchical organization and that neoplastic cells derive from the clonal expansion and aberrant growth of a limited subset of stem cells designated as cancer stem cells (CSC) [4]. CSCs share important features with normal tissue stem cells including self-renewal and ability to differentiate into the heterogeneous non-tumorigenic cells that represent the bulk of a tumor. CSCs are believed to be the only cells with tumor-initiation ability within cancer tissues and they would be responsible to initiate both primary tumors as well as recurrences and metastases [4]. According to this model, targeting of CSC is essential for cancer cure. However, CSC has been reported to be relatively refractory to traditional therapies that mainly target the rapidly dividing cells that constitute the majority of the non-stem cell component of tumors. This property of CSC could explain why anti-cancer therapies often fail and why cancer recurrences represent a frequent event in several cancer patients including OSCC whose poor prognosis is mainly due to a low response rate to the available antineoplastic drugs.

Putative CSCs can be identified based on the expression of specific cell surface markers [4] and CD133 has proven to be most useful for the identification of CSCs in a variety of solid tumors [4]. Human CD133 (PROM1, prominin-1, AC133 antigen) is a cell surface glycoprotein originally identified as the target of the AC133 monoclonal antibody developed for its ability to react with normal CD34+ hematopoietic stem cells $[4,5]$. CD133 expression has been detected in a variety of normal as well as cancer stem cells in several human tissues including

*Corresponding author: Alessandro Sgambato, MD, Ph.D., Istituto di Patologia Generale, Università Cattolica del Sacro Cuore, Largo Francesco Vito 1, 00168 Rome, Italy, Tel: 39-06-3016619; Fax: 39-06-3012753; E-mail: asgambato@rm.unicatt.it

Received February 08, 2014; Accepted March 17, 2014; Published March 20 2014

Citation: Reggiani Bonetti L, Migaldi M, Boninsegna A, Fanali C, Farina M, et al (2014) Expression of CD133 Correlates with Tumor Stage, Lymph Node Metastasis and Recurrence in Oral Squamous Cell Carcinoma. J Cancer Sci Ther 6: 094-098. doi:10.4172/1948-5956.1000255

Copyright: (c) 2014 Reggiani Bonetti L, et al. This is an open-access article distributed under the terms of the Creative Commons Attribution License, which permits unrestricted use, distribution, and reproduction in any medium, provided the original author and source are credited. 
Citation: Reggiani Bonetti L, Migaldi M, Boninsegna A, Fanali C, Farina M, et al. (2014) Expression of CD133 Correlates with Tumor Stage, Lymph Node Metastasis and Recurrence in Oral Squamous Cell Carcinoma. J Cancer Sci Ther 6: 094-098. doi:10.4172/1948-5956.1000255

in human OSCC [6-9] and animal model of oral cancer [10]. It is noteworthy that CD133 expressing cells have been shown to display an increased resistance to therapy thus supporting the hypothesis that they might play an important role in determining the clinical behavior of OSCC [7].

In the present study, we analyzed by immunostaining the expression of the CD133 molecule in a large series of oral cancers and evaluated its relation with traditional prognostic indicators and with the clinical outcome of the patients. The results obtained suggest that the CD133 immunohistochemical evaluation can be useful in the management of OSCC patients. The implications of these findings are discussed.

\section{Materials and Methods}

\section{Samples}

Tissue specimens were obtained from 68 Italian patients with cancer of the oral cavity who underwent surgery at the University Hospital of Modena, Italy during the period 1995-2007. No patient received pre-operative chemotherapy or radiotherapy. Formalin fixed, paraffin embedded specimens were retrieved from the archive of the Pathology Institute and two experienced pathologists (MM and LRB) confirmed the histological diagnosis of each lesion. The study was approved by the Research Ethics Committee of the University of Modena and Reggio Emilia, Modena, Italy.

All cases were classified according to WHO criteria [11]. Staging and grading were defined according to the International Union Against Cancer [12]. The series included 43 (63\%) males and 25 (37\%) females with a mean age at diagnosis of 62.6 years (range, 26 to 93 years) and a mean follow-up of 41.70 months (range, 14 to 170 months).

\section{Immunohistochemistry}

Immunohistochemical analyses were performed on routinely processed, formalin-fixed, paraffin-embedded tissues employing an avidin-biotin complex immunoperoxidase technique (Vectastain $A B C$ kit, Vector Laboratories, Burlingame, CA), as previously described [13-15]. Briefly, successive $5 \mu \mathrm{m}$ tissue sections were cut from blocks selected for the presence of representative lesions and mounted on charged and precleaned slides. Sections were dewaxed, rehydrated and then microwave pretreated $(10 \mathrm{~min}$ at $750 \mathrm{~W}$ in $1 \mathrm{mM}$ EDTA buffer, $\mathrm{PH}$ 8.0) for antigen retrieval. A specific polyclonal anti-CD133 antibody (Santa Cruz Biotechnology, Santa Cruz, CA, USA; 1:100) was used for the staining and was applied overnight at $4^{\circ} \mathrm{C}$. Comparable results but with a weaker staining were obtained using the monoclonal AC133 antibody (Miltenyi Biotec, Bergisch Gladbach, Germany; 1:10) (data not shown). Only a clear staining of the cell membrane and/or cytoplasm was regarded as positive.

Controls for specificity of staining were performed by immunostaining duplicate sections in the absence of the primary antibody. A specimen with known positive immunostaining for CD133 served as a positive control. Positive and negative control slides were included within each batch of slides. The fraction of positive cells was scored by examining at least 10 random high-power fields $(\mathrm{x} 400)$ for each sample and the percentage of cells with positive reaction was calculated semi-automatically by means of a computer-assisted cellular image analyzer (Image-Pro Plus, Media Cybernetics, Silver Spring, MA, USA), as previously reported [13-15].

All scoring and interpretations of the results were made by two of the authors independently (MM and LRB) without knowledge of other clinical-pathological variables. To assess interobserver variation, the results of the two measurements were compared by paired t test and no statistical differences were found (data not shown). The few cases with discrepant scoring were re-evaluated jointly on a second occasion, and agreement was reached.

\section{Statistical analysis}

The association between CD133 expression and other molecular and clinical-pathological parameters were calculated using contingency table methods and tested for significance using the Fisher exact test (two-sided). Comparison of mean was performed using ANOVA means test. Patients were uniformly followed and disease free survival (DFS) was defined as the interval between surgery and the first documented evidence of disease in local-regional area and/or distant sites. Overall survival was defined as the interval between surgery and death from the disease. Disease free and overall survival curves were calculated using the Kaplan-Meier method and the log-rank test was used to compare survival curves. Univariate and multivariate relative risks were calculated using the Cox proportional hazards regression. All calculations were performed using the SPSS (Statistical Package for the Social Sciences rel. 13) software (Chicago, IL, USA) and the results were considered statistically significant when the $p$ value was $<0.05$.

\section{Results}

To investigate the role of CD133 in human oral carcinogenesis, the expression of CD133 was evaluated by immunostaining in a series of 68 consecutive primary human oral squamous cell carcinomas (OSCC).

In OSCC immunostaining was frequently heterogeneous within one specimen, both in terms of percent of positive cells and intensity (Figure 1). The median percentage of positive cells was 5 (range 0-80; mean $=11$ ) and the CD133 staining was not detectable in tumor cells in $29(43 \%)$ cases. No correlation was observed with age at diagnosis, gender and grading (Table 1).

On the other hand, a significant association was observed with tumor stage (pT) and nodal status (N). In fact, grouping together pT3

\begin{tabular}{|c|c|c|c|c|c|c|}
\hline \multirow[b]{3}{*}{ Gender } & \multirow[t]{3}{*}{ Total } & \multirow{2}{*}{\multicolumn{2}{|c|}{$\begin{array}{c}\text { Negative } \\
\mathbf{n}(\%)\end{array}$}} & \multirow{2}{*}{\multicolumn{2}{|c|}{$\begin{array}{c}\text { Positive } \\
\text { n (\%) }\end{array}$}} & \multirow[t]{3}{*}{$p$ value } \\
\hline & & & & & & \\
\hline & & & & & & \\
\hline Males & 43 & 18 & (42) & 25 & (58) & \\
\hline Females & 25 & 11 & (44) & 14 & (56) & n.s. \\
\hline \multicolumn{7}{|l|}{ Age (yr) } \\
\hline$\leq 62^{\circ}$ & 34 & 11 & (32) & 23 & (68) & \\
\hline$>62$ & 34 & 18 & (53) & 16 & (47) & n.s. \\
\hline \multicolumn{7}{|c|}{ Tumor Grading } \\
\hline 1 & 22 & 8 & (36) & 14 & (64) & \\
\hline 2 & 27 & 13 & $(48)$ & 14 & (52) & \\
\hline 3 & 19 & 8 & (42) & 11 & (58) & ns \\
\hline \multicolumn{7}{|c|}{ Tumor Stage } \\
\hline pT1 & 10 & 6 & $(60)$ & 4 & $(40)$ & \\
\hline pT2 & 15 & 10 & (66) & 5 & (34) & \\
\hline pT3 & 6 & 1 & (17) & 5 & (83) & \\
\hline pT4 & 37 & 12 & (32) & 25 & (68) & 0.04 \\
\hline \multicolumn{7}{|c|}{ Nodal status } \\
\hline Negative & 30 & 18 & $(60)$ & 12 & $(40)$ & \\
\hline Positive & 38 & 11 & (29) & 27 & (71) & 0.01 \\
\hline
\end{tabular}

${ }^{\circ}$ median value

Table 1: CD133 expression in relation to clinic-pathological parameters in a series of 68 oralsquamous cell carcinoma patients. 
Citation: Reggiani Bonetti L, Migaldi M, Boninsegna A, Fanali C, Farina M, et al. (2014) Expression of CD133 Correlates with Tumor Stage, Lymph Node Metastasis and Recurrence in Oral Squamous Cell Carcinoma. J Cancer Sci Ther 6: 094-098. doi:10.4172/1948-5956.1000255

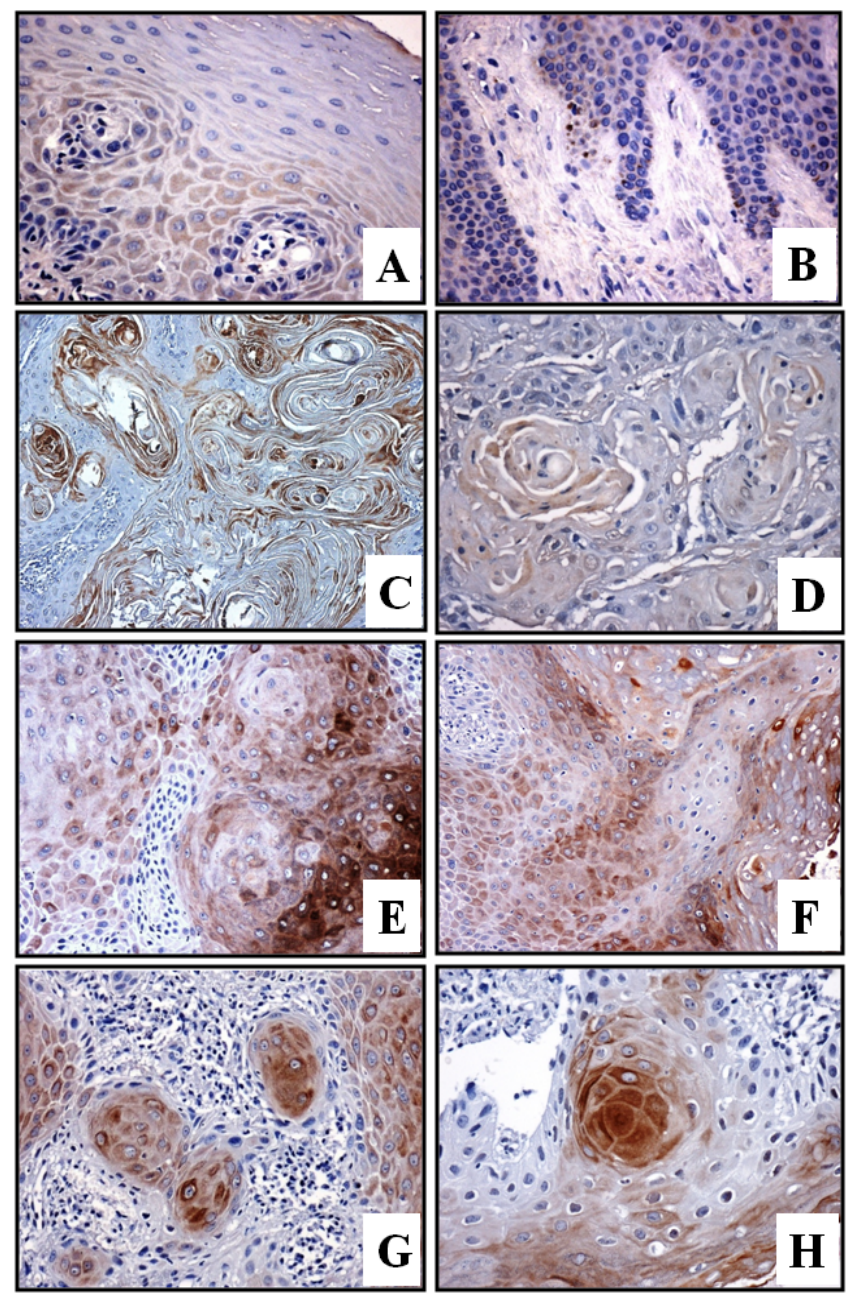

Figure 1: Examples of immune histochemical staining for CD133 in normal oral epithelium (A, B) and OSCC (C-H). (A) Normal squamous epithelium displaying a diffuse basal and para-basal immunopositivity (x200). (B) Normal squamous epithelium with immunopositivity localized to the basal layer (x150) (C) A well-differentiated OSCC with a diffuse immunopositivity (x100). (D) A moderately differentiated OSCC with a focal immunopositivity (x200). (E-H) Different OSCC samples with an intense immunopositivity for CD133, localized primarily in epithelial nests infiltrating surrounding stroma (x200).

and pT4 (advanced) vs pT1 and pT2 (early) tumors, positive staining for CD133 was detected in 9 (36\%) of the 25 pT1-2 tumors and in $30(70 \%)$ of the 43 advanced pT3-4 cancers and cross-tab analysis identified a significant correlation $(\mathrm{p}<0.04)$ between the two parameters $($ Table 1). Similarly, positive staining for CD133 was detected in $12(40 \%)$ of the N-negative cancers and in $27(71 \%)$ of the tumors with nodal involvement and this difference was significant $(\mathrm{p}<0.01)$ (Table 1).

When CD133 staining was analyzed in relation with clinical outcome, median disease free survival (DFS) of CD133 negative patients was longer compared to positive cases $(43 \pm 40$ vs $27 \pm 21$, months) and this difference showed a trend toward significance ( $\mathrm{p}=$ 0.07).

Moreover, when tumors were stratified according with CD133 expression, three (10\%) out of 29 negative cases and 14 (36\%) among the remaining 39 cases recurred during the period of follow-up and this difference was significant $(\mathrm{p}<0.012)$ as also confirmed by the Kaplan-
Meier curves of DFS which displayed a significant separation between the two groups of patients ( $\mathrm{p}=0.001$ by log-rank test) (Figure $2 \mathrm{~A}$ ).

On the other hand, median disease overall survival (OS) was 40 $($ mean $=49.41)$ and 31 (mean $=35.87)$ months, respectively, for CD133 negative and CD133 positive patients and this difference was not significant $(\mathrm{p}=0.09)$. Moreover, stratifying tumors according with CD133 expression, 11 (38\%) out of 29 negative cases and 17 (44\%) among the remaining 39 cases died of the disease during the period of follow-up but this difference was not significant as also confirmed by the Kaplan-Meier curves of OS which did not display a significant separation between the two groups of patients (Figure 2B).

Univariate analyses of other clinical-pathological parameters (i.e., grade, stage and nodal status) confirmed a prognostic significance both in terms of DFS and OS for both stage and nodal status but not for tumor grade which did not show any relationship with clinical outcome (Figures $2 \mathrm{C}-\mathrm{F}$ and data not shown).

In a multivariate analysis performed by building a Cox hazards model that included tumor stage and CD133, only higher tumor stage but not CD133 staining confirmed to be an independent predictor of shorter disease-free and overall survival (Table 2). Nodal status was not included in the model because of its collinearity with tumor stage and results did not change including lymph node metastasis and/or tumor grade in the model (data not shown).

\section{Discussion}

In this study, expression of the CD133 protein was evaluated by immunohistochemistry in a series of OSCC. As previously mentioned, oral cancer is the most frequent cancer of the head and neck region and is becoming a pressing problem with oral squamous cell carcinomas (OSCC) accounting for about $90 \%$ of cases [1]. Prognostic evaluation for OSCC is mainly based on clinical TNM classification, but this staging system is not sufficient to accurately predict clinical outcome in single patients [2]. Thus, a biomarker that could help to predict the prognosis of OSCC patients would be a valuable tool for clinicians and would allow a better prognostic and therapeutic stratification of OSCC patients.

To our knowledge, this is the first report analysing by immunostaining CD133 expression in a large subset of OSCC and evaluating its relation with the clinical outcome of patients. We observed an heterogeneous staining for CD133 in OSCC samples and CD133 was not detectable in tumor cells in $29(43 \%)$ cases. No correlation was observed with age at diagnosis, gender and grading

\begin{tabular}{|c|c|c|c|}
\hline & Risk & $95 \%$ confidence & \\
\hline Variable & ratio & interval & $p$ value \\
\hline \multicolumn{4}{|c|}{ DISEASE-FREE SURVIVAL } \\
\hline Age $^{*}$ & 1.487 & $0.421-4.247$ & 0.458 \\
\hline Tumor stage\# & 14.692 & $1.871-115.370$ & 0.001 \\
\hline CD133** & 2.632 & $0.685-10.121$ & 0.159 \\
\hline \multicolumn{4}{|c|}{ OVERALL SURVIVAL } \\
\hline Age $^{*}$ & 2.625 & $1.161-5.931$ & 0.020 \\
\hline Tumor stage\# & 5.993 & $2.030-17.691$ & 0.001 \\
\hline CD133** & 1.063 & $0.469-2.409$ & 0.513 \\
\hline
\end{tabular}

*The risk ratio is given as older versus younger patients. \#The risk ratio is given as higher versus lower stage tumors. ${ }^{* *}$ The risk ratio is given as positive versus negative tumors

Table 2: Contribution of various potential prognostic factors to overall survival by Cox regression analysis in oral squamous cell carcinoma patients. 
Citation: Reggiani Bonetti L, Migaldi M, Boninsegna A, Fanali C, Farina M, et al. (2014) Expression of CD133 Correlates with Tumor Stage, Lymph Node Metastasis and Recurrence in Oral Squamous Cell Carcinoma. J Cancer Sci Ther 6: 094-098. doi:10.4172/1948-5956.1000255

but a significant association was observed with tumor stage and lymph node metastasis (Table 1). Positive staining for CD133 also displayed a significant relationship with high risk of disease progression in univariate analysis while no relationship was observed with cancer specific survival (Figure 2). In a multivariate analysis, CD133 staining did not confirm to be an independent predictor of shorter survival compared with more traditional parameters such as tumor stage and lymph node metastasis (Table 2).

As previously mentioned, CD133 is presently considered a useful marker to identify CSC in a variety of human cancers, including head and neck and oral cancer [6-10]. and it is expected to identify cells which have the ability to initiate tumor growth and are also the major responsible of tumor recurrence and progression including colonization of distant organs and metastasis [9-16]. The results of the present study are partially in agreement with a previous paper reporting a significantly higher expression of CD133 in stage III to IV than in stage I to II OSCC in the absence of a significant correlation with age, sex, location of tumor and lymph node metastasis [17]. It is of interest that in the latter study CD133 was not detectable in twenty (38\%) of the 52 OSCC analyzed, a finding consistent with the lack of CD133 staining observed in 29 (43\%) cases of the present study and suggesting that $\mathrm{CD} 133^{+}$cells might be lost during OSCC progression and/or that other cell surface markers might identify CSC in a subset of OSCC [18]. However, the significant relationship between CD133 expression and tumor stage supports an important role of CD133expressing cells in the development and progression of OSCCs and is consistent with data supporting a pivotal role of CD133 in stemness properties and tumorigenesis of head and neck SCC cells (16) as well as in driving malignant transformation of oral lichen planus [19] and oral leukoplakia [20].
The results of the present study are also in agreement with previous studies suggesting a prognostic significance of CD133 immunohistochemical evaluation in several types of cancer patients [21]. as well as with the observation that CD133-expressing cells are associated with increased resistance to chemotherapy [7] which might explain the worse outcome of CD133-expressing cancers. In multivariate analyses performed by building a Cox hazards model including all the factors found to be associated with disease progression in the univariate analyses, positive CD133 staining did not confirm to be an independent predictor of worse prognosis compared to tumor stage and nodal status (Table 2). Available data and the results of the present study confirm that tumor stage can be considered a useful parameter for the identification of high risk OSCC patients (Figure 2). However, it is not always capable to predict disease evolution in the single patients. Thus, the availability of a prognostic marker which could be performed directly on the surgical tumor specimen using a simple, immunohistochemical technique appears as an extremely useful and promising factor for the identification of OSCC patients at high risk of disease progression who might benefit of an adjuvant therapy following surgical resection. Moreover, it is noteworthy that there is a great interest for the development of therapies specifically targeting CSC whose elimination is considered essential for the eradication of malignancies and for the development of curative antineoplastic treatments approaches [22]. Therapeutic approaches specifically targeting CD133 expressing cells have been already proposed and proved promising against oral cancer cells [23]. Thus, immunohistochemical analysis of CD133 expression might also be helpful to select patients suitable for treatment specifically targeting CD133+ cells. The introduction of CD133 immunostaining in the clinical practice, however, is not expected to be easily achieved. In fact, it will be important to reach a consensus agreement on the antibody to
A



B

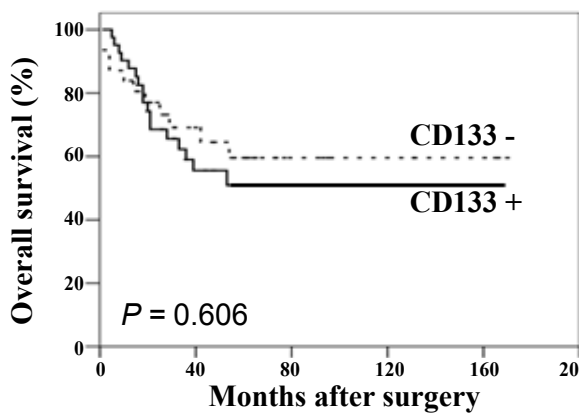

C

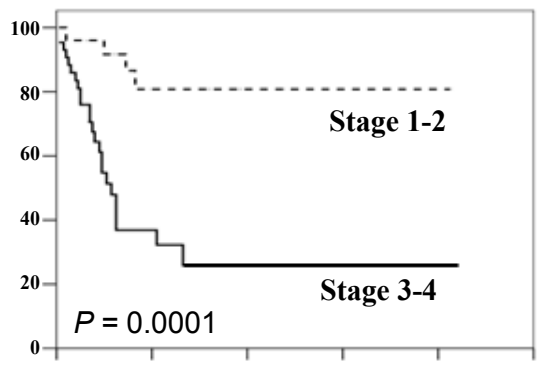

D

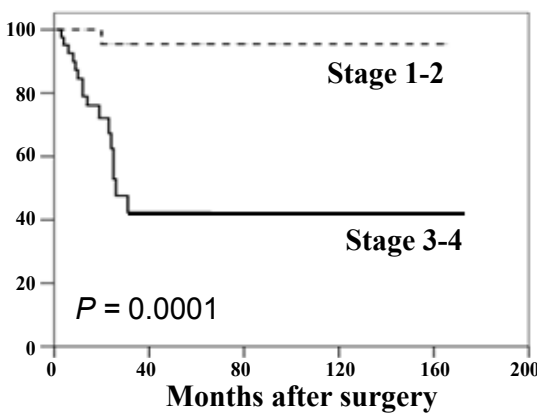

$\mathbf{E}$

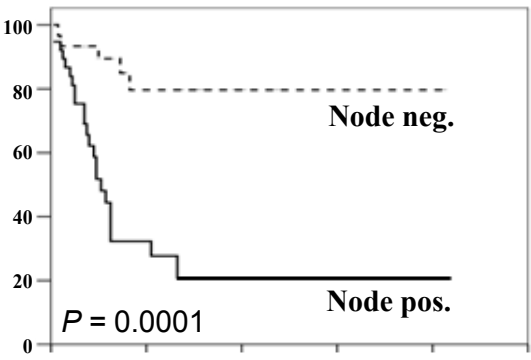

$\mathbf{F}$

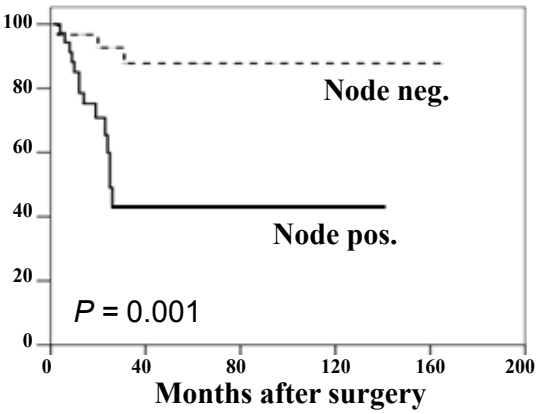

Figure 2: Kaplan-Meier curves for disease-free (A, C, E) and overall (B, D, F) survival in OSCC patients. Patients were stratified by CD133 expression (A, B), tumor stage $(C, D)$ and nodal status $(E, F)$. 
Citation: Reggiani Bonetti L, Migaldi M, Boninsegna A, Fanali C, Farina M, et al. (2014) Expression of CD133 Correlates with Tumor Stage, Lymph Node Metastasis and Recurrence in Oral Squamous Cell Carcinoma. J Cancer Sci Ther 6: 094-098. doi:10.4172/1948-5956.1000255

be used for the analysis as well as the criteria for the evaluation of the staining, since different approaches have been reported in the literature [24].

In conclusion, we investigated CD133 expression in a subset of OSCC cancer patients and demonstrated a correlation of its expression with other prognostic indicators as well as with the clinical outcome of patients in terms of disease-free survival. These findings suggest that CD133 might represent a useful prognostic marker for OSCC patients and warrant additional studies on a larger series of cases to further elucidate the role of CD133 in the development and progression of OSCC and its suitability as a prognostic biomarker predictive of cancer aggressiveness and patient outcome. If confirmed on larger cohorts of patients, these findings could have therapeutic implications by helping to identify patients at higher risk of progression and death from the disease who would benefit most from a systemic adjuvant treatment after surgical resection. Moreover, CD133 might well be a candidate molecular target for the development of new therapeutic interventions if a direct role of the molecule in the process of tumor development and progression will be demonstrated in oral tumorigenesis.

\section{Acknowledgments}

This work was supported in part by grants from the Università Cattolica to A.S. from the Italian Association for Cancer Research (AIRC) and the Emilia-Romagna Region.

\section{References}

1. Siegel R, Naishadham D, Jemal A (2013) Cancer statistics, 2013. CA Cancer J Clin 63: 11-30.

2. Mydlarz WK, Hennessey PT, Califano JA (2010) Advances and Perspectives in the Molecular Diagnosis of Head and Neck Cancer. Expert Opin Med Diagn 4: 53-65.

3. Massano J, Regateiro FS, Januário G, Ferreira A (2006) Oral squamous cell carcinoma: review of prognostic and predictive factors. Oral Surg Oral Med Oral Pathol Oral RadiolEndod 102: 67-76.

4. Vermeulen L, de Sousa e Melo F, Richel DJ, Medema JP (2012) The developing cancer stem-cell model: clinical challenges and opportunities. Lancet Oncol 13 : e83-89.

5. Miraglia S, Godfrey W, Yin AH, Atkins K, Warnke R, et al. (1997) A novel fivetransmembrane hematopoietic stem cell antigen: isolation, characterization, and molecular cloning. Blood 90: 5013-5021.

6. Okamoto A, Chikamatsu K, Sakakura K, Hatsushika K, Takahashi G, et al (2009) Expansion and characterization of cancer stem-like cells in squamous cell carcinoma of the head and neck. Oral Oncol 45: 633-639.

7. Zhang Q, Shi S, Yen Y, Brown J, Ta JQ, et al. (2010) A subpopulation of CD133(+) cancer stem-like cells characterized in human oral squamous cell carcinoma confer resistance to chemotherapy. Cancer Lett 289: 151-160.

8. Felthaus O, Ettl T, Gosau M, Driemel O, Brockhoff G, et al. (2011) Cancer stem cell-like cells from a single cell of oral squamous carcinoma cell lines. BiochemBiophys Res Commun 407: 28-33.
9. Zhang Z, Filho MS, Nör JE (2012) The biology of head and neck cancer stem cells. Oral Oncol 48: 1-9.

10. Pang LY, Bergkvist GT, Cervantes-Arias A, Yool DA, Muirhead R, et al. (2012) Identification of tumour initiating cells in feline head and neck squamous cell carcinoma and evidence for gefitinib induced epithelial to mesenchymal transition. Vet J 193: 46-52.

11. World Health Organization (2005) International Classification of Diseases Edited by Geneva, Switzerland, IARC press.

12. AJCC (American Joint Committee on Cancer) (2002) Cancer Staging Manual. Edited by Greene F, Page D, Fleming I, Fritz A, Balch C, Haller D, Morrow M. New York, USA, Springer - Verlag.

13. Arena V, Caredda E, Cufino V, Stigliano E, Scaldaferri F, et al. (2011) Differential CD133 expression pattern during mouse colon tumorigenesis. Anticancer Res 31: 4273-4275.

14. ReggianiBonetti L, Migaldi M, Caredda E, Ponz de Leon M, Di Gregorio C, et al. (2012) Increased expression of CD133 is a strong predictor of poor outcome in stage I colorectal cancer patients. Scand J Gastroenterol 47: 1211-1217.

15. Coco C, Zannoni GF, Caredda E, Sioletic S, Boninsegna A, et al. (2012) Increased expression of CD133 and reduced dystroglycan expression are strong predictors of poor outcome in colon cancer patients. J Exp Clin Cancer Res 31: 71.

16. Chen YS, Wu MJ, Huang CY, Lin SC, Chuang TH, et al. (2011) CD133/SrC axis mediates tumor initiating property and epithelial-mesenchymal transition of head and neck cancer. PLoS One 6: e28053.

17. Ravindran G, Devaraj H (2012) Aberrant expression of CD133 and musashi-1 in preneoplastic and neoplastic human oral squamous epithelium and their correlation with clinicopathological factors. Head Neck 34: 1129-1135.

18. Sinha N, Mukhopadhyay S, Das DN, Panda PK, Bhutia SK (2013) Relevance of cancer initiating/stem cells in carcinogenesis and therapy resistance in oral cancer. Oral Oncol 49: 854-862.

19. Sun L, Feng J, Ma L, Liu W, Zhou Z (2013) CD133 expression in oral lichen planus correlated with the risk for progression to oral squamous cell carcinoma. Ann DiagnPathol 17: 486-489.

20. Liu W, Wu L, Shen XM, Shi LJ, Zhang CP, et al. (2013) Expression patterns of cancer stem cell markers ALDH1 and CD133 correlate with a high risk of malignant transformation of oral leukoplakia. Int J Cancer 132: 868-874.

21. Grosse-Gehling P, Fargeas CA, Dittfeld C, Garbe Y, Alison MR, et al. (2013) CD133 as a biomarker for putative cancer stem cells in solid tumours: limitations, problems and challenges. J Pathol 229: 355-378.

22. Schulenburg A, Ulrich-Pur H, Thurnher D, Erovic B, Florian S, et al. (2006) Neoplastic stem cells: a novel therapeutic target in clinical oncology. Cancer 107: $2512-2520$.

23. Damek-Poprawa M, Volgina A, Korostoff J, Sollecito TP, Brose MS, et al. (2011) Targeted inhibition of CD133+ cells in oral cancer cell lines. J Dent Res 90: 638-645.

24. Sgambato A, Errico F, Caredda E, Puglisi MA, Cittadini A (2011) Divergen expression of CD133 in different studies: the need for a consensus panel? Int J Cancer 128: 2247-2249. 